\title{
Lung lobes and fissures: a morphological study
}

\author{
Ajay Ratnakarrao Nene, Krishna Swami Gajendra, Manchiraju Venkata Ramananda Sarma \\ Department of Anatomy, GSL Medical College, Andhra Pradesh, India
}

\begin{abstract}
Objectives: Variations of lobar pattern and fissures of lung have been described on imaging techniques whereas there are fewer studies in gross anatomy. Present study was performed to examine lung specimens with respect to the morphology of fissures and lobes, to note the variations, to compare them with previous studies and to find their clinical implications.

Methods: Fifty pairs of lungs were used for the study, obtained from 50 formalin-fixed adult Indian cadavers. The lung specimens were meticulously observed for the patterns of lobes and fissures, variations were noted and specimens were photographed.

Results: Seven right lungs showed absence of horizontal fissure while one specimen showed absent oblique fissure. On the left side 6 specimens showed incomplete oblique fissure. In addition, 2 right-sided specimens showed presence of superior accessory fissure whereas the inferior accessory fissure was observed in 7 right and 12 left-sided specimens. The left minor fissure was seen in 13 specimens and one pair of lungs exhibited fissures separating all four segments seen on diaphragmatic surface.

Conclusion: The results and their comparison with the previous works show that there is a wide range of difference in occurrence of major, minor and accessory fissures between and among different populations. Knowledge of such variations might explain bizarre presentation of certain clinical cases pertaining to lung pathologies. Also knowing the frequency of occurrence of a variant fissure in a particular population might help the radiologist and clinician to make correct diagnosis, plan, execute and modify a surgical procedure depending on the merit of the case.
\end{abstract}

Key words: accessory fissure; horizontal fissure; lung; oblique fissure

Anatomy 2011;5:30-38, (c) 2011 TSACA

\section{Introduction}

Amongst the pair of lungs in the thoracic cavity, the right lung is broader and heavier than the left lung. Two fissures oblique and horizontal (Figure 1), divide it into three lobes namely, upper, middle and lower. The oblique fissure separates the lower lobe from the remaining two lobes. It runs obliquely and crosses the inferior border of the lung about $7.5 \mathrm{~cm}$ behind its anterior end. The horizontal fissure separates the upper and middle lobe. It begins from the oblique fissure, runs horizontally and cuts the anterior border at inner end of fourth costal cartilage. The longer and lighter left lung is divided into a superior and an inferior lobe by an oblique fissure (Figure 1) which extends from costal to medial sur- faces of the lung both above and below the hilum. It begins on the medial surface posterosuperior to the hilum, runs obliquely upwards and backward to cut the posterior border of the lung about $6 \mathrm{~cm}$ below the apex and then passes downward and forward across the costal surface. The more vertical left oblique fissure is approximately indicated by vertebral border of scapula in fully abducted arm. ${ }^{[1]}$

Finding accessory fissures in lung specimens is not uncommon, but appreciating them on radiographs and CT scans is difficult and hence they are either not appreciated as distinct entities or are completely misinterpreted. They usually occur at the boundaries between bronchopulmonary segments. ${ }^{[2]}$ The commonly found acces- 
Figure 1. Oblique and horizontal fissures in the lung. $(\mathbf{a}, \mathbf{b})$ costal surface; (c, d) medial surface.

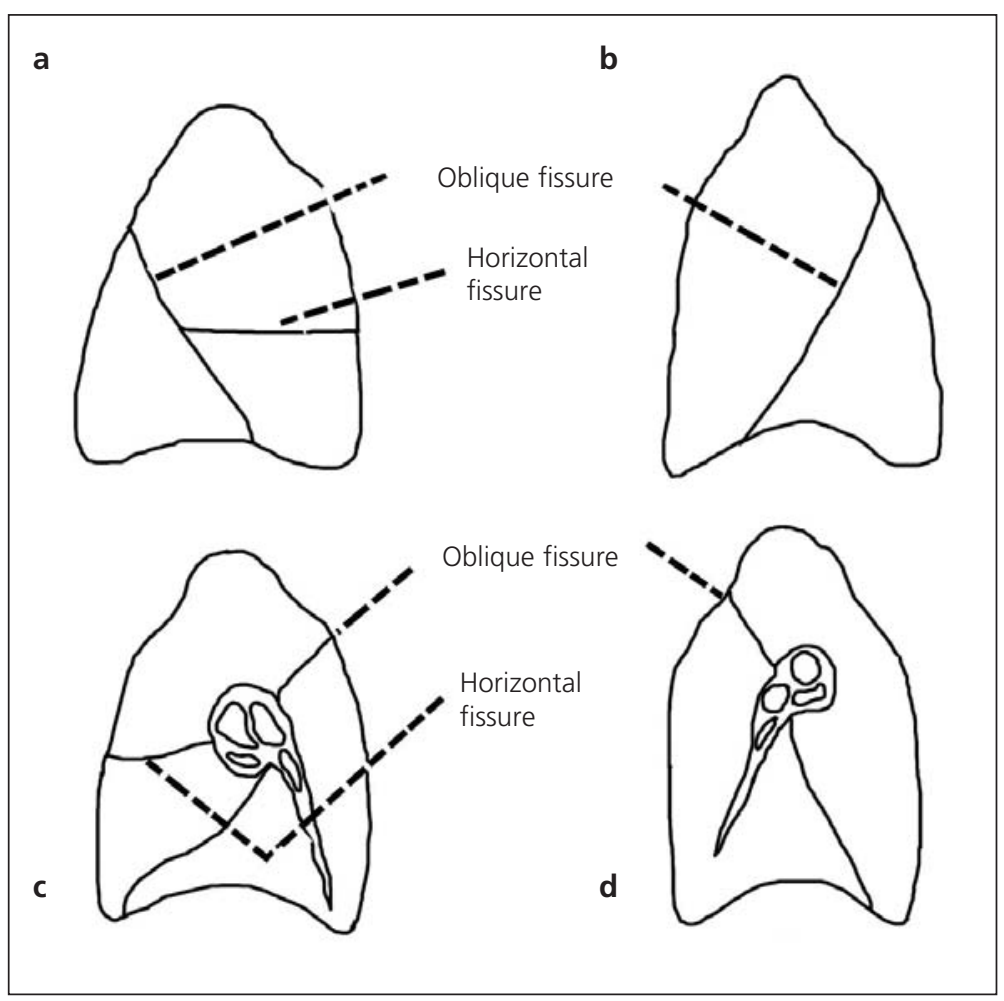

sory fissures are superior accessory fissure, inferior accessory fissure and left minor fissure (Figure 2). The superior accessory fissure separates superior segment from the rest of the segments of lower lobe of lung, the inferior accessory fissure separates a small 'infracardiac lobe' from other segments of lower lobe of lung on the diaphragmatic surface and the left minor fissure separates the lingula from the other segments of upper lobe of left lung. ${ }^{[2]}$

The fissures may vary in the degree of completeness and tend to divide the lobe into smaller subdivisions (Figure 2b). Complete fissures show continuity of lobes at their bottom only by the parts of bronchial tree and blood vessels. In the region of incomplete fissures the adjacent lobes are connected by a sizeable chunk of pulmonary tissue as the cleft fails to reach the hilum. The fissure may be absent altogether (Figures $\mathbf{2 c}, \mathbf{d}$ and $\mathbf{3}$ ). The fissures are conducive to uniform expansion of lobes. They provide routes for movements of lobes in relation to each other. This is more relevant to distension and movement of the lower lobes during breathing. ${ }^{[3]}$

The appearance of accessory lung fissure varies on $\mathrm{X}$ ray and CT scan. This is seen especially in reference to depth or completeness of the fissure. A fissure appearing complete on $\mathrm{X}$-ray might be seen as an incomplete one on CT scan and vice versa may also be seen. ${ }^{[4]}$
Certain radiological findings are specific enough to clinch the diagnosis as in the cases of azygos lobe. In the presence of azygos lobe, the CT scan shows a significant increase in the size of mediastinum in front of and behind the trachea. Another important finding seen is a significant chunk of lung tissue in front of superior vena cava. ${ }^{[5]}$

The knowledge of anatomical variations of lobes of the lung is important for identifying precise location, extent and morphology of bronchopulmonary segments. Many times the radiologists may misinterpret them on an X-ray or a CT scan. Anatomical knowledge of such variations is useful for planning lobectomies and surgical resections involving individual segment.

Hence this study aims to find variations in the morphology of lung fissures and lobes in the Indian population and comparing the findings with previous studies to derive a conclusion. This might serve to generate some new data regarding lung lobes and fissures which can be made available to the radiologists and surgeons to help in making correct diagnosis and appropriate planning of surgery.

\section{Materials and Methods}

Fifty pairs of lungs from equal number of both sexes of formalin-fixed Indian cadavers used for undergraduate 


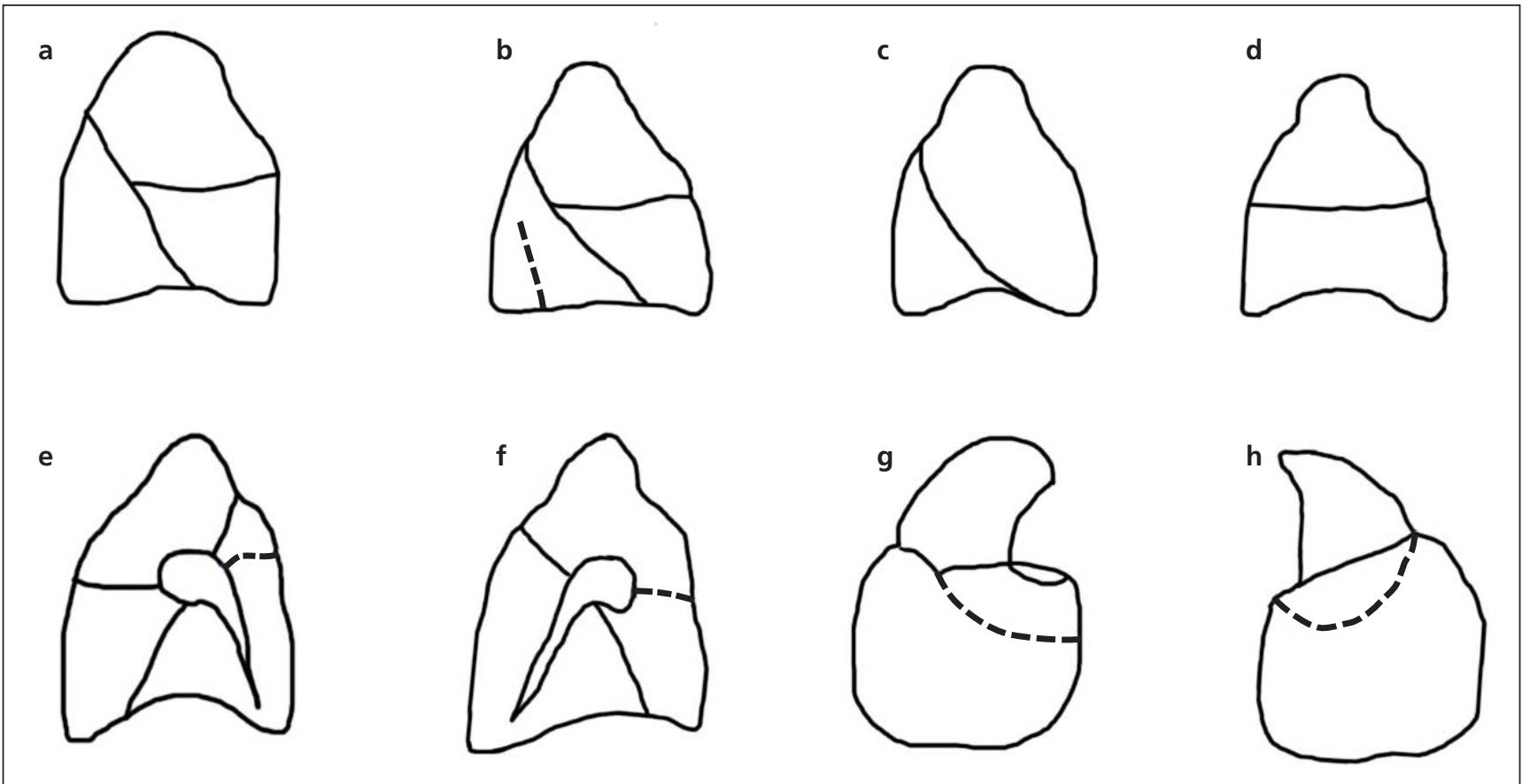

Figure 2. Variant major, minor and accessory fissures (dotted lines) as seen in the present study. (a-d) lateral surface of right lung; (e, f) medial surface of left lung; ( $\mathbf{g}, \mathbf{h}$ ): inferior surface. (a) normal anatomy; (b) subdivision of lower lobe; (c) absent horizontal fissure; (d) absent oblique fissure; (e) superior accessory fissure; (f) left minor fissure; $(\mathbf{g}, \mathbf{h})$ inferior accessory fissure.

dissection classes were used for this study. Details of morphology of lobes and fissures; presence of any variant fissure, accessory fissure were recorded. The speci- mens were photographed by a digital camera (Sony Cybershot; Model - W220; 12.1 Megapixel with 4x optical zoom).

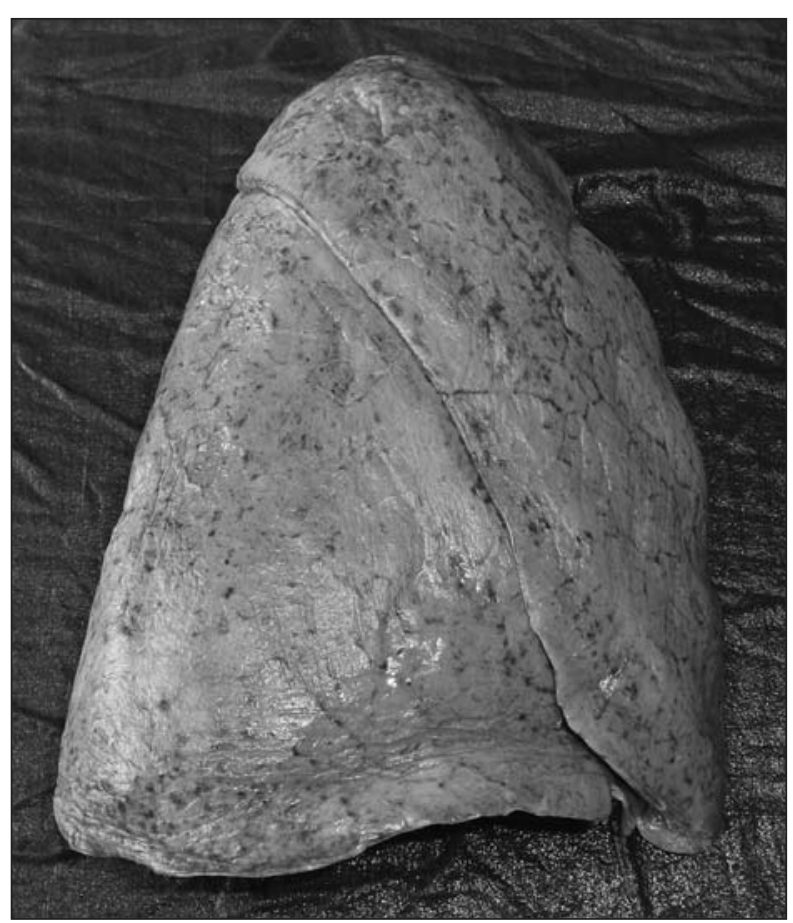

Figure 3. Absent horizontal fissure of right lung seen from costal surface. 


\section{Results}

The observations regarding incidence of major (oblique) and minor (horizontal) lung fissures are tabulated in Table 1.

\section{Right lung specimens}

The horizontal fissure was absent in seven right-sided lungs (14\%) and hence middle lobe was not appreciated (Figure 2c). The horizontal fissure was incomplete in 8\% of lungs and oblique fissure was incomplete in $6 \%$ of lungs and hence the lobation was imperfect. In $2 \%$ of the specimens, the oblique fissure was absent (Figure 2d). Totally, out of 50 right lungs, thirty-four lungs (68\%) exhibited the normal pattern of fissures and lobes (Figure 2a). Nine lungs (18\%) out of 50, showed presence of accessory fissures. One specimen showed separation of four bronchopulmonary segments visible on diaphragmatic surface, by formation of accessory fissures. ments visible on diaphragmatic surface, by formation of accessory fissures.

\section{Accessory fissures}

The observations regarding accessory fissures of the lungs are tabulated in Table 2 . The table shows incidence of accessory fissures was more on the left side than right side cumulatively. The superior accessory fissure (SAF) occurred in $4 \%$ of cases in right lung while it was not seen at all in left lung. The inferior accessory fissure (IAF) was found in $14 \%$ and $24 \%$ of specimens of right side and left sides, respectively. The left minor fissure (LMF) was seen in $26 \%$ of lung specimens.

The observations regarding incidence of major and minor lung fissures according to Craig and Walker's criteria are tabulated in Table 3.

Craig and Walker ${ }^{[6]}$ have proposed a manner of classification of fissure for describing operative technique

Table 1

Comparison of different studies indicating the variations of major and minor fissures

\begin{tabular}{|c|c|c|c|c|c|c|}
\hline & Fiss & e parameter & Present study & Meenakshi et al..$^{[9]}$ & IEHAV ${ }^{[10]}$ & Lukose et al. ${ }^{[8]}$ \\
\hline \multirow{4}{*}{ Right lung } & \multirow{2}{*}{$\begin{array}{r}\text { Oblique } \\
\text { fissure }\end{array}$} & Absent & $2 \%$ & - & - & - \\
\hline & & Incomplete & $6 \%$ & $36.6 \%$ & $30 \%$ & - \\
\hline & \multirow{2}{*}{$\begin{array}{r}\text { Horizontal } \\
\text { fissure }\end{array}$} & Absent & $14 \%$ & $16.6 \%$ & $21 \%$ & $10.5 \%$ \\
\hline & & Incomplete & $8 \%$ & $63.3 \%$ & $67 \%$ & $21 \%$ \\
\hline \multirow{2}{*}{ Left lung } & \multirow{2}{*}{$\begin{array}{r}\text { Oblique } \\
\text { fissure }\end{array}$} & Absent & $0 \%$ & - & - & - \\
\hline & & Incomplete & $12 \%$ & $46.6 \%$ & $30 \%$ & $21 \%$ \\
\hline
\end{tabular}

IEHAV: Illustrated Encyclopedia of Human Anatomical Variations.

\section{Left lung specimens}

The oblique fissure was incomplete in $12 \%$ of specimens and 29 out of 50 left-sided lungs (58\%), exhibited the normal pattern of fissures and lobes. Twenty specimens (40\%) showed presence of accessory fissure. One specimen showed separation of four bronchopulmonary seg-

Table 2

Incidence of accessory fissures

\begin{tabular}{lcc}
\hline Accessory fissure & Right lung & Left lung \\
\hline SAF & $4 \%$ & $0 \%$ \\
IAF & $14 \%$ & $24 \%$ \\
LMF & - & $26 \%$ \\
\hline
\end{tabular}

SAF: superior accessory fissure; IAF: inferior accessory fissure; LMF: left minor fissure. and also for comparing different surgical series. The criteria used to classify the lung fissures were degree of completeness of fissure and the location of the pulmonary artery at the base of the oblique fissure. Four grades have been described: Grade I- complete fissure with entirely separate lobes; Grade II- complete visceral cleft but parenchymal fusion at the base of the fissure; Grade III- visceral cleft evident for a part of the fissure; and Grade IV- complete fusion of lobes with no evident fissure line. From the data in this study, morphological variations involving the oblique fissure in $58 \%$ of leftsided lungs, $70 \%$ of right-sided lungs and variations involving the horizontal fissure in $68 \%$ of right-sided lungs can be classified as Grade I. Similarly, variations involving the horizontal fissure in $12 \%$ of right-sided lungs and variations involving the oblique fissure in $22 \%$ 
Table 3

Incidence of major and minor fissures of lungs according to Craig and Walker's criteria

\begin{tabular}{rrrccc}
\hline \multirow{2}{*}{ Lung } & Fissure & Grade I & Grade II & Grade III & Grade IV \\
\hline \multirow{2}{*}{ Right lung } & Horizontal & $68 \%$ & $12 \%$ & $6 \%$ & $14 \%$ \\
\cline { 2 - 6 } & Oblique & $70 \%$ & $22 \%$ & $6 \%$ & $2 \%$ \\
\hline \multirow{2}{*}{ Left lung } & Oblique & $58 \%$ & $30 \%$ & $12 \%$ & $0 \%$ \\
\hline
\end{tabular}

of right-sided lungs and 30\% left-sided lungs can be categorized as Grade II. Additionally, Grade III fissures were seen in $6 \%$ and $12 \%$ of oblique fissures of right and left lungs, respectively. Horizontal fissure in $14 \%$ and oblique fissure in $2 \%$ of right-sided lung were found to be absent (Grade IV).

The observations regarding incidence of accessory fissures of lung according to Craig and Walker's criteria are tabulated in Table 4.

Amongst the accessory fissures, Grade II SAF were found in $4 \%$ of right lung specimens, whereas $2 \%$ Grade I and $12 \%$ Grade III IAF were found in the right lung specimens. On the left side 4\% Grade II and 20\% Grade III IAF were seen while the three LMF seen were all Grade II. No SAF was observed in any of the left lung specimens.

\section{Discussion}

During the developmental stage, the lung tissue grows as multiple bronchopulmonary buds. Later the spaces or fissures that separate individual bronchopulmonary buds / segments become obliterated. The spaces remain along the interlobar planes to give rise to major (oblique) and minor (horizontal) fissures in a fully developed lung. ${ }^{[7]}$ Absence or incompleteness of a fissure could be due to obliteration of these fissures either completely or partially. On the other hand, accessory fissure could be the result of non-obliteration of spaces which normally are obliterated. Incomplete pulmonary fissures indicating partial fusion between lobes are common and more than half of the pulmonary fissures are incomplete.

When compared with the studies by Lukose et al., ${ }^{[8]}$ Meenakshi et al., ${ }^{[9]}$ and Bergman and Afifi, ${ }^{[10]}$ a lower incidence of variations was observed in all the parameters used in the present study, whereas oblique fissure was found to be absent in $2 \%$ of cases which was not mentioned in previous studies.

In the study of Lukose et al. ${ }^{[8]}$ incomplete and absent horizontal fissure was reported in $21 \%$, and $10.5 \%$, respectively. Incomplete oblique fissure was present in $21 \%$ of left-sided lungs. In the present study, incomplete and absent horizontal fissure was seen in $8 \%$, and $14 \%$ of cases, respectively. Incomplete oblique fissure was seen in $12 \%$ of left lungs (Table 1).

Accessory fissures of the lung are commonly observed in lung specimens, but are often unappreciated or misinterpreted on radiographs and CT scans. They usually occur at the boundaries between bronchopulmonary segments. Accessory fissures can be mistakenly confused with areas of linear atelectasis, pleural scars, or walls of bullae. ${ }^{[2]}$

\section{Inferior accessory fissure (IAF)}

The inferior accessory fissure is seen around the medial basal segment of the lower lobe (Figures $2 \mathrm{~g}$, $\mathrm{h}$ and 4 ). On the diaphragmatic surface of the lung, the fissure

Table 4

Incidence of accessory fissures of lungs according to Craig and Walker's criteria

\begin{tabular}{rrcccc}
\hline \multirow{2}{*}{ Lung } & Accessory Fissure & Grade I & Grade II & Grade III & Grade IV \\
\hline \multirow{2}{*}{ Right lung } & SAF & 0 & 4 & 0 & 0 \\
\cline { 2 - 6 } & IAF & 2 & 0 & 12 & 0 \\
\hline \multirow{3}{*}{ Left lung } & SAF & 0 & 0 & 0 & 0 \\
& IAF & 0 & 4 & 20 & 0 \\
& LMF & 0 & 6 & 20 & 0 \\
\hline
\end{tabular}

SAF: superior accessory fissure; IAF: inferior accessory fissure; LMF: left minor fissure 
Figure 4. Basal surface of left lung showing inferior accessory fissure (arrows) separating an infracardiac lobe.

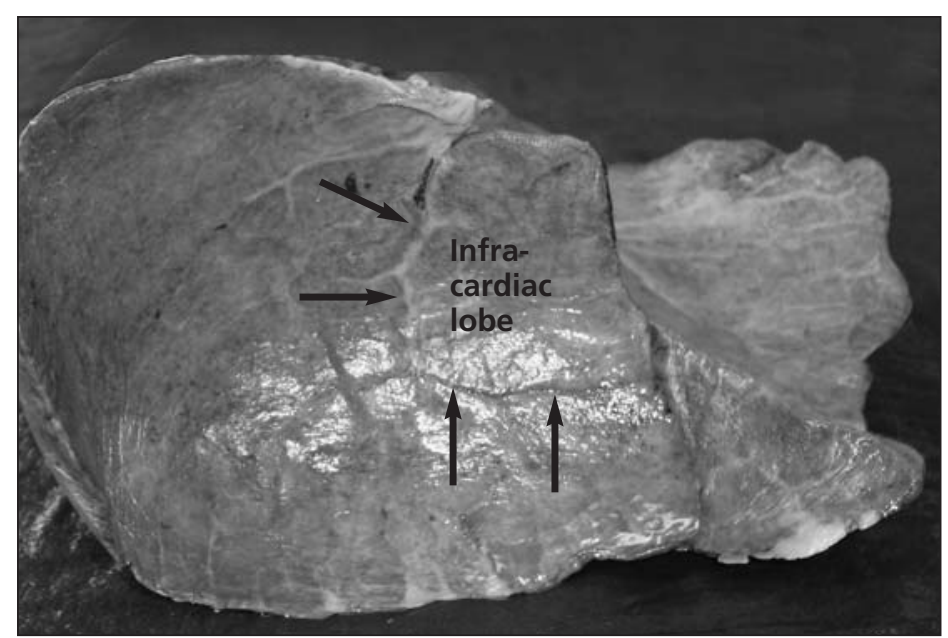

extends laterally from the region near the pulmonary ligament and then makes a convex arch forward to join the major fissure. ${ }^{[2]}$

\section{Superior accessory fissure (SAF)}

The superior accessory fissure is seen in the territory of lower lobe (Figures 2e and 5). It partially or completely separates the superior segment of the lower lobe from the basal segments. When it is present, the superior segment has been called the posterior or dorsal lobe. The fissure varies in length and depth from a complete fissure to a notch. ${ }^{[2]}$

Figure 5. Right lung showing an incomplete superior accessory fissure (SAF), seen from costal surface.

\section{Left minor fissure (LMF)}

A left minor fissure separates the lingula from the rest of the left upper lobe (Figures $\mathbf{2} \mathbf{f}$ and $\mathbf{6}$ ). In almost all cases, the usual segmental anatomy of the left lung is preserved. ${ }^{[2]}$

On CT scans, accessory fissures are seen as high attenuation curvilinear band. ${ }^{[11]}$ Muller et al. ${ }^{[12]}$ in their radiological study observed that the SAF was rarely seen on radiographs and CT scans while the IAF could be seen on approximately $8 \%$ of chest radiographs. In the present study, the SAF was seen in $4 \%$ and $0 \%$ of lungs on right

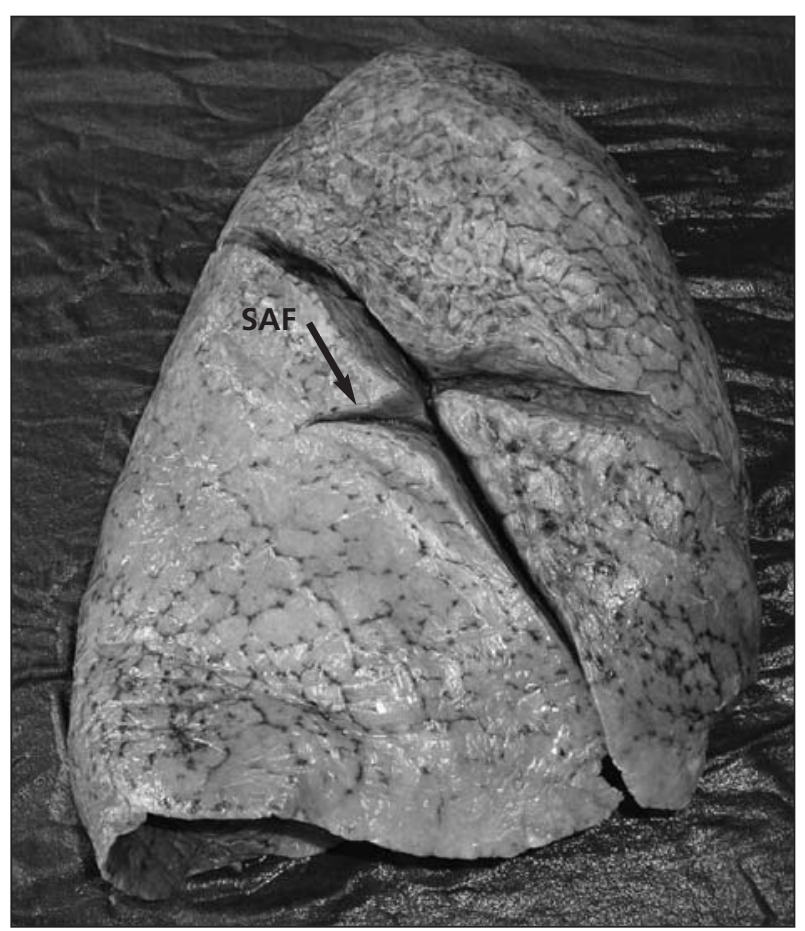




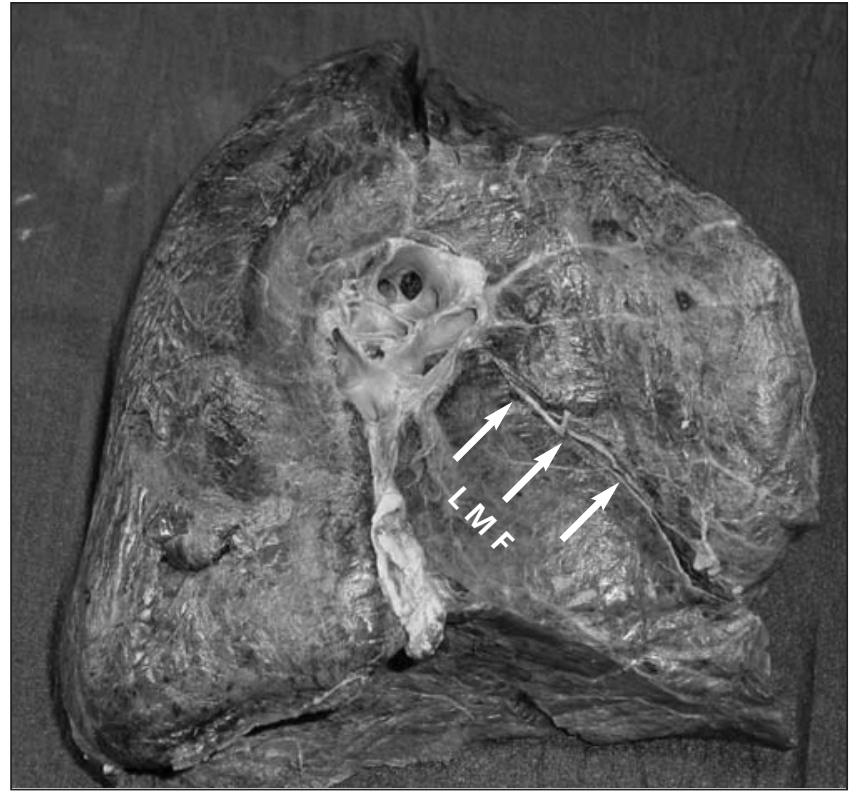

and left sides, respectively and IAF was found in 14\% and $24 \%$ on right and left sides, respectively (Table 2 ).

Godwin and Tarver ${ }^{[2]}$ in their extensive study commented that IAF was present in $40 \%-50 \%$ of specimens. The incidence of SAF in their anatomic specimens ranged from $5 \%$ of left lower lobes to $14 \%$ on the left, $30 \%$ on the right, and $12 \%$ bilaterally. Both the fissures varied in length, depth and degree of completeness. In the present study, the SAF was observed in $4 \%$ of right lung specimens, while the IAF was seen in $14 \%$ and $24 \%$ of specimens of right and left sides, respectively.

Gesase, ${ }^{[13]}$ in a study of 102 lung specimens, found incidence of LMF to be $10.78 \%$, incomplete horizontal fissure as $7.84 \%$, and diaphragmatic fissure to be $7.84 \%$. Further $2.94 \%$ of left lungs had three lobes while $5.88 \%$ of right lungs had two lobes. In the present study, the incidence of LMF was found to be $26 \%$, incomplete horizontal fissure was seen in $8 \%$ of lungs and diaphragmatic fissure was observed in $14 \%$ of right and $24 \%$ of left lungs. The right lung showed two lobes in $16 \%$ of cases while the left lung showed three lobes in $6 \%$ of cases in the present study.

Cronin et al. ${ }^{[14]}$ in their study of 150 consecutive multi-detector CT scan (MDCT) examinations found that $100 \%$ of subjects had oblique fissure on both sides, $96.7 \%$ of subjects showed right horizontal fissure. Forty percent subjects had accessory fissures and $16 \%$ of subjects showed presence of LMF. In the present study, the oblique fissure was found to be present in $98 \%$ of lungs and right horizontal fissure was seen in $86 \%$ of speci-
Figure 6. Medial surface of left lung showing left minor fissure (LMF) (arrows).

mens. Eighteen percent of right lung specimens and $40 \%$ of left lung specimens showed presence of accessory fissures in the present study, the maximum incidence was that of LMF $26 \%$. This implies there is increased incidence of accessory fissures found in the present study compared with the study of Cronin et al. ${ }^{[14]}$

Aziz et al.$^{[15]}$ in their study of 622 patients performed high-resolution CT scan (HRCT) from aortic arch to diaphragm and concluded that oblique fissures were seen facing laterally in their upper part in $100 \%$ of right and $89 \%$ of left lungs. The right oblique fissure was incomplete in $48 \%$ of cases and the left oblique fissure was incomplete in $43 \%$ of cases. The accessory fissures as seen were azygos fissure, IAF, SAF and LMF in $1.2 \%$, $8.6 \%, 4.6 \%$ and $8.1 \%$ of cases, respectively. In the present study, the right oblique fissure was incomplete in $6 \%$ and left oblique fissure was incomplete in $12 \%$ of cases. SAF was observed in $4 \%$ of right lung specimens, while the IAF was seen in $14 \%$ and $24 \%$ of specimens of right and left sides, respectively. The incidence of LMF was found to be $26 \%$ (Table 2).

Berkmen et al..$^{[4]}$ in their imaging study of 17 patients by CT scan and X-ray found 18 accessory fissures. They observed disparity in the appearance of fissures in X-ray and CT scan. On the basis of configuration of the accessory fissure on CT scan, they classified the fissures into four types (Types I, II, III and IV). Type I fissure ran parallel to the chest wall and was convex outwards. It was found in 8 cases. Type II fissure ran parallel to sagittal plane and was convex inwards. It was seen in 2 cases. Type III fissure was represented by a straight or curved 
linear shadow running anteromedially. It was observed in 5 cases. Type IV fissure ran parallel to same sided oblique fissure between mediastinum and chest wall. It was found in 3 cases.

In the present study the accessory fissures were classified into four grades according to Craig and Walker's criteria. The percentage incidence of Grade I fissure was found to be $2 \%$, Grade II fissure was seen in $14 \%$ of specimens, Grade III fissure in 52\% and Grade IV fissure in $0 \%$ (Table 4).

Austin, ${ }^{[16]}$ in his study of LMF in 2000 consecutive chest radiographs, observed that LMF was not encountered very often in X-rays. He reported an incidence of 32 cases in 2000 subjects radiographed. He observed that LMF was seen as a curved line convex upwards with lateral end higher than the medial. Rarely the fissure was horizontal. In the present study LMF stood out to be the most frequently occurring accessory fissure seen in $26 \%$ of specimens.

The fissures in the lung help even expansion of lung tissue and serve as a reliable landmark on the lung. Accessory fissures when present at abnormal locations in the lungs give rise to abnormal lobes of the lung aerated by normal bronchus. Such finding is more common in infants. ${ }^{[3]}$ From a radiological point of view, an accessory fissure may commonly be misinterpreted as a lung lesion. ${ }^{[17]}$ In patients with endobronchial lesion, an accessory fissure might alter the usual pattern of lung collapse and pose difficulty in diagnosing a lesion and its extent by giving an unusual appearance. An incomplete major fissure causes the odd appearance of fluid tracking within the fissure. Incomplete fissures may also alter the spread of disease within the lung. Pneumonia in a particular lobe is contained within the confines of the lobe by complete and normal fissure. In patients with incomplete fissures, pneumonia may spread to adjacent lobes through the parenchymal continuation. Odd lobar involvement with carcinoma of the lung may be explained on a similar basis. ${ }^{[18]}$

Many diseases require accurate segmental localization of the lesion and the knowledge of accessory fissure is of much clinical importance to the clinician. Preoperative planning and strategy for segmental resection or pulmonary lobectomy may also change during presence of such accessory fissure.

An incomplete fissure is also a cause for postoperative air leakage. Often these accessory fissures act as a barrier to spread of infection, creating a sharply marginated pneumonia which can wrongly be interpreted as atelectasis or consolidation. ${ }^{[2]}$
The knowledge of anatomy of fissures of lung may help clarifying confusing radiographic findings like extension of fluid into an incomplete major fissure or spread of various diseases through different pathways. ${ }^{[19]}$

\section{Conclusion}

The results of present study and their comparison with the previous works show that there is a wide range of difference in occurrence of major, minor and accessory fissures between and among different populations. This implies that a variety of genetic and environmental factors might affect development of these fissures. Knowledge of such variations might explain bizarre presentation of certain clinical cases pertaining to lung pathologies.

Also knowing the frequency of occurrence of a variant fissure in a particular population might help the radiologist and clinician to make correct diagnosis. Similarly, it might help the surgeon to plan, execute and modify a surgical procedure depending on the merit of the case. This will help to reduce the morbidity and mortality associated with lung surgeries.

\section{References}

1. Shah P, Johnson D, Standring S. Thorax. In: Gray's Anatomy. 39th ed. Edinburgh: Churchill Livingstone; 2005. p. 1068-9.

2. Godwin JD, Tarver RD. Accessory fissures of the lung. AJR Am J Roentgenol 1985;144:39-47.

3. Rosse C, Gaddum-Rosse P. Hollinshead's Textbook of Anatomy. Philadelphia: Lipincott-Raven; 1997. p. 441-61.

4. Berkmen T, Berkmen YM, Austin JH. Accessory fissures of the upper lobe of the left lung: CT and plain film appearance. AJR Am J Roentgenol 1994;162:1287-93.

5. Speckman JM, Gamsu G, Webb WR. Alterations in CT mediastinal anatomy produced by an azygos lobe. AJR Am J Roentgenol 1981;137:47-50

6. Craig SR, Walker WS. A proposed anatomical classification of the pulmonary fissures. J R Coll Surg Edinb 1997;42:233-4.

7. Larsen WJ. Human Embryology. New York: Churchill Livingstone; 1993. p. 111-30.

8. Lukose R, Paul S, Sunitha DM, et al. Morphology of the lungs: variations in the lobes and fissures. Biomedicine 1999;19:227-32.

9. Meenakshi S, Manjunath KY, Balasubramanyam V. Morphological variations of the lung fissures and lobes. Indian J Chest Dis Allied Sci 2004;46:179-82.

10. Bergman RA, Afifi AK, Miyauchi R. Variations in peripheral segmentation of right lung and the base of the right and left lungs. In: Illustrated Encyclopedia of Human Anatomic Variation. http://www.anatomyatlases.org/AnatomicVariants/OrganSystem/ Text/LungsTrachea.shtml (28th of November, 2010). 
11. Butler P, Mitchell AWM, Ellis H. Applied Radiological Anatomy. Cambridge (UK): Cambridge University Press; 1999. p. 136-8.

12. Muller NL, Fraser RS, Colman NC, Pare PD. The normal chest. In: Radiologic Diagnosis of Diseases of Chest. Philadelphia: WB Saunders Company; 2001. p. 25-6.

13. Gesase AP. The morphological features of major and accessory fissures observed in different lung specimens. Morphologie 2006;90: 26-32.

14. Cronin P, Gross BH, Kelly AM, Kazerooni EA, Carlos RC. Normal and accessory fissures of the lung: evaluation with contiguous volumetric thin-section multidetector CT. Eur J Radiol 2010;75:e1-8.
15. Aziz A, Ashizawa K, Nagaoki K, Hayashi K. High resolution CT anatomy of the pulmonary fissures. J Thorac Imaging 2004;19: 186-91.

16. Austin JHM. The left minor fissure. Radiology 1986;161:433-6.

17. Aldur MM, Denk CC, Çelik HH, Taşçıŏlu AB. An accessory fissure in the lower lobe of the right lung. Morphologie 1997;81:5-7.

18. Tarver RD. How common are incomplete pulmonary fissures, and what is their clinical significance? AJR Am J Roentgenol 1995;164: 761.

19. Dandy WE. Incomplete pulmonary interlobar fissure sign. Radiology 1978;128:21-5.

Correspondence to: Ajay Ratnakarrao Nene, Associate Professor Department of Anatomy, GSL Medical College, NH-5, Lakshmipuram, Rajahmundry, Dist - East Godavari, Andhra Pradesh, Pin- 533296, India Phone: 088324041 67; Fax: 08832483023 e-mail: ajay.nene@gmail.com Conflict of interest statement: No conflicts declared. 